Meta

Journal des traducteurs

Translators' Journal

\title{
Le rôle des mots d'emprunt dans la traduction biblique
}

\section{Jean-Claude Margot}

Volume 35, numéro 1, mars 1990

Actes du colloque international " La traduction proligère "

URI : https://id.erudit.org/iderudit/003574ar

DOI : https://doi.org/10.7202/003574ar

Aller au sommaire du numéro

Éditeur(s)

Les Presses de l'Université de Montréal

ISSN

0026-0452 (imprimé)

1492-1421 (numérique)

Découvrir la revue

Citer cet article

Margot, J.-C. (1990). Le rôle des mots d'emprunt dans la traduction biblique. Meta, 35(1), 188-194. https://doi.org/10.7202/003574ar d'utilisation que vous pouvez consulter en ligne.

https://apropos.erudit.org/fr/usagers/politique-dutilisation/ 


\section{LE RÔLE DES MOTS D'EMPRUNT DANS LA TRADUCTION BIBLIQUE}

JEAN-ClaUde MARGoT

Alliance biblique universelle, Aubonne, Suisse

Selon le Dictionnaire de linguistique de Jean Dubois et al. (Paris 1973: 188), «il y a emprunt linguistique quand un parler A utilise et finit par intégrer une unité ou un trait linguistique qui existait précédemment dans un parler B et que A ne possédait pas; l'unité ou le trait emprunté sont eux-mêmes appelés emprunts. L'emprunt est le phénomène socio-linguistique le plus important dans tous les contacts de langue (...), c'est-à-dire d'une manière générale toutes les fois qu'il existe un individu apte à se servir totalement ou partiellement de deux parlers différents (...).» Ce phénomène socio-linguistique si important paraît parfaitement normal à certains, mais profondément inquiétant pour d'autres. Et ce n'est pas dans le contexte québécois qu'il est nécessaire d'en dire beaucoup plus sur les raisons de cette inquiétude!

Sans vouloir jouer les arbitres dans la controverse qui existe à ce sujet, j'aimerais, en ce qui me concerne, établir tout d'abord une distinction: 1. D'une part, il faut tenir compte du fait que la tendance à l'emprunt est inséparable de l'évolution des langues, de sorte que, comme le dit le Dictionnaire cité plus haut, «contrairement à une opinion assez répandue, [cette tendance] n'est pas le lot exclusif des temps modernes» (ibid.). À cet égard, je ne saurais mieux faire que de renvoyer ici à l'ouvrage de Claude Hagège, $L e$ Français et les siècles, Paris, 1987, qui, en opposition à certaines réactions passionnelles, décrit le phénomène d'une façon équilibrée et solidement fondée du point de vue linguistique (voir en particulier les pages 55 et ss., ainsi que le paragraphe intitulé «La vocation d'emprunt», pp. 75 et ss.). Il dit par exemple ceci :

Même s'il est vrai que cette évolution, inscrite dans la nature des langues, peut passer par d'autres voies que l'emprunt, ce dernier y tient une place très importante. Le mot étranger pénètre avec la chose lorsque celle-ci appartient à un environnement technique ou culturel trop nouveau pour qu'un mot autochtone la traduise adéquatement. Cela vaut pour toutes les langues. Cela vaut donc pour le français (...) il ne saurait y avoir de langue pure d'apports étrangers. Pas plus qu'il n'y a de race pure (p. 78 ).

2. Mais, d'autre part, le phénomène de l'emprunt peut aussi être envisagé sous un angle plus particulier, à savoir celui de la traduction. Et là, on ne saurait le considérer simplement comme allant de soi. Je pense spécialement aux traducteurs qui, parce qu'ils ont de la peine à trouver dans la langue d'arrivée l'équivalent exact d'un terme ou d'une expression de la langue-source, jugent naturel de recourir à l'emprunt; il en résulte souvent qu'ils sont obligés de mettre en bas de page une note donnant le sens du mot employé, que les lecteurs ignorent. Dans de nombreux cas, il s'agit d'une solution de facilité, condamnable par là même. Cependant, le procédé n'est pas forcément blâmable; il est parfois tout à fait justifié. On en vient donc à se demander: quels sont les critères permettant de juger si un emprunt est légitime ou non? Et c'est là le problème que j'aimerais traiter à l'aide d'exemples empruntés au domaine de la traduction biblique.

$\mathrm{Si}$, dans un colloque consacré à la traduction en général, j'aborde ce sujet à partir de la traduction biblique, c'est que, me semble-t-il, on n'a jamais autant recouru à 
l'emprunt que dans les nombreuses versions bibliques, en quelque langue que ce soit. (On peut mettre à part la traduction scientifique, car il s'agit là avant tout d'harmoniser la terminologie internationale plutôt que de limiter les emprunts.) Par conséquent, le champ d'expérience est très étendu dans ce type de traduction et les leçons à en tirer très instructives.

\section{RÉACTION CONTRE UNE TRADITION BIEN ETABLIE}

Les versions françaises de la Bible contiennent un certain nombre de mots empruntés au grec ou au latin, qui ont passé dans l'usage sinon courant, du moins ecclésiastique. Ce fait avait déjà frappé les auteurs d'une nouvelle traduction du Nouveau Testament, parue à Lausanne (Suisse) en $1839^{1}$, au point qu'ils ont voulu réagir contre cette tradition séculaire. L'un d'eux s'en est expliqué dans un ouvrage rédigé quelques années plus $\operatorname{tard}^{2}$. Il cite en particulier des mots grecs latinisés qu'on a introduits dans les Bibles françaises, mais qui, estime-t-il, sont mal compris: ainsi Évangile, apôtre, église, évêque, diacre ou synagogue. Il y a également des mots empruntés directement au latin, comme résurrection, ressusciter, rédemption, rémission, enfer, gentil, tabernacle, homme, animal ou encore charité. Or, les traducteurs de la nouvelle version ont voulu rendre plus clairement le sens de ces mots en disant, par exemple, Bonne Nouvelle pour Évangile, envoyé pour apôtre, assemblée pour église, congrégation pour synagogue (au sens d'assemblée) ou serviteur pour diacre, en ce qui concerne les termes empruntés au grec. Quant à ceux venant du latin, on a remplacé résurrection, ressusciter par réveil et relèvement, réveiller et se réveiller, relever et se relever, ou rédemption par rachat, pour ne donner que deux exemples ${ }^{3}$.

La tentative de ces traducteurs du siècle passé est tout à la fois intéressante et contestable. Intéressante en ce sens qu'elle attire l'attention sur le risque de malentendu qu'entraîne un emprunt inconsidéré à une autre langue (fût-elle de la même famille), en raison d'une déviation de sens ou de l'évolution du vocabulaire. Dans le cas d'un des mots cités ci-dessus, évêque, il est évident que sa signification dans le Nouveau Testament n'est pas celle qu'il prendra plus tard dans l'Église, à partir du II $\mathrm{I}^{\mathrm{e}}$ siècle de notre ère. De même, plusieurs expériences m'ont prouvé que le mot rémission, au sens de pardon, n'est plus guère compréhensible que pour des gens ayant étudié le latin.

Mais la démarche de ces traducteurs est en même temps contestable sur plus d'un point. En effet, elle ne tient pas suffisamment compte d'un critère ayant une valeur capitale lorsqu'il s'agit de juger de l'opportunité ou de la non-opportunité d'un emprunt: je veux parler de son rapport avec l'usage. Ainsi, dans le contexte ecclésiastique actuel, une «assemblée chrétienne» désigne autre chose qu'une «Église chrétienne»: cette seconde expression, d'un usage d'ailleurs très courant, comporte en particulier un aspect institutionnel qui est en général absent de la première.

Les deux éléments que je viens de relever, à savoir risque de déviation et relation à l'usage, méritent d'être précisés en raison de leur importance pour le sujet qui nous occupe. C'est pourquoi je vais leur consacrer les deux prochaines sections.

\section{EMPRUNT ET GLISSEMENT DE SENS}

Partons tout d'abord d'une constatation d'ordre général. La déviation ou le glissement de sens est un phénomène bien connu des spécialistes qui ont étudié la question de l'emprunt. Dans un ouvrage collectif intitulé Linguistique, publié en $1980^{4}$, Frédéric François présente quelques exemples suggestifs à cet égard. Ils sont tirés de la thèse d'un Zaïrois, Musanji Ngalasso Mwata, consacrée aux emprunts romans en langue pende du Zaïre. On y constate que les termes empruntés aux langues européennes subissent des modifications de sens que l'on peut classer en quatre catégories : 
a) restrictions: ainsi lâbe (du français «abbé») utilisé pour désigner un prêtre noir, tandis que le prêtre européen a droit à mâpe (de «mon père»);

b) extensions: ainsi bândi («bandit») appliqué à toute personne turbulente;

c) déplacements valorisant ou dévalorisant: ainsi lêso (de «leçon») qui a pris le sens de mensonge!

d) déplacements métonymiques: ainsi lâméle (de «la mer»), qui n'exprime pas l'idée de mer, mais celle de poisson.

Or, on constate des phénomènes tout à fait semblables dans l'histoire de la traduction biblique. Pour illustrer cette affirmation, voici un premier exemple concernant la déviation de sens: dans la Bible hébraïque, on trouve un verbe, paqad, qui, appliqué à Dieu, désigne souvent son intervention soit dans un sens positif («intervenir en faveur de»), soit dans un sens négatif («intervenir pour juger, punir»). La Vulgate latine 1'a rendu par visitare, verbe qui peut convenir dans certains contextes, puisqu'il signifie «voir souvent, visiter, inspecter, éprouver, affliger ${ }^{5} »$. En revanche, lorsque la plupart des versions françaises traduisent systématiquement «paqad» par «visiter», sous l'influence du latin, il y a perte de sens: le texte biblique n'annonce pas une simple visite (de courtoisie), au sens actuel du terme, de la part de Dieu, mais, comme nous venons de le voir, son intervention. Dans ce cas, on se soumet sans hésitation à une tradition terminologique remontant à la Vulgate de saint Jérôme, et cela aussi bien du côté protestant que du côté catholique. J'entendais un jour un prédicateur citer le texte de LuC 1,68, «Béni soit le Seigneur (...) parce qu'il a visité son peuple», en précisant que le verbe en cause signifie «intervenir en faveur (de son peuple)», mais, ajoutait-il, «littéralement: visiter»! En fait, tel n'est pas le sens littéral du verbe employé, même en grec comme ici. Il y aurait beaucoup à dire quant à la façon abusive, chez bon nombre de biblistes, de donner le sens soidisant littéral des mots, dans la traduction ou en note! Toutefois, je sortirais des limites de mon sujet en abordant ce thème maintenant ${ }^{6}$.

Je viens de faire allusion au rôle joué par la tradition terminologique dans le domaine des versions bibliques. De telles traditions peuvent soulever des problèmes très difficiles à résoudre lorsqu'on entreprend des traductions dites interconfessionnelles, c'est-à-dire entre protestants et catholiques, comme c'est le cas dans de nombreuses régions du monde. À ce propos, il me suffira de mentionner ce que j'ai vu au Rwanda il n'y a pas longtemps: les traducteurs de la nouvelle version du Nouveau Testament en kinyarwanda, la principale langue du pays, ont eu à affronter de nombreuses difficultés de cet ordre? Par exemple, les catholiques utilisaient traditionnellement le mot Kiliziya pour «Église», emprunté au latin ecclesia, lui-même emprunté au grec ekklêsia. Le comité de traduction voulait écarter Kiliziya, en raison même de son origine étrangère. De leur côté, les protestants employaient itorero, un terme connu en kinyarwanda, certes, mais auquel les catholiques attribuent une connotation sectaire, car, dans l'usage actuel, il se rapporte à diverses dénominations autres que l'Église catholique. Par ailleurs, pour «prêtre», les catholiques avaient umusacerdoti, où l'on reconnât facilement l'emprunt au latin sacer$d o s$, avec adjonction du préfixe umu propre à la classe humaine. Cette situation est typique, non seulement parce qu'elle montre à quel point il peut être difficile de résoudre de tels problèmes terminologiques, mais aussi parce qu'elle prouve que le recours à l'emprunt, qui peut paraitre satisfaisant sur le moment, risque de se révéler aléatoire par la suite en raison de connotations imprévisibles au départ.

Toujours en relation avec la question de la déviation ou de la restriction de sens, voici un autre exemple qui nous vient des îles Samoa. La langue de ces îles a des affinités avec celle d'autres parties de la Polynésie, Tahiti en particulier. À l'époque où a débuté la traduction de la Bible dans cette région (première moitié du XIX ${ }^{\mathrm{e}}$ siècle), le pain y était inconnu. C'est pourquoi les traducteurs ont emprunté au grec le terme artos, «pain», qui, 
adapté à la structure phonologique et orthographique de la langue, est devenu areto ${ }^{8}$. Cependant, ultérieurement, le pain a fait son apparition dans ces îles; les gens se sont mis à en consommer et l'ont appelé «falaoa», à partir d'un emprunt à l'anglais «flour», farine. Il en est résulté que la parole de Jésus «Je suis le pain de vie» (Jean 6, 35) a perdu son véritable impact, dans la mesure où Jésus faisait allusion au pain de la vie courante, tandis que areto, mot conservé dans le texte biblique, n'est plus compris que comme une allusion au pain de l'Eucharistie. Dans ce cas-là, on est à cheval sur deux questions : celle de la restriction de sens et celle de l'usage (qui sera traitée dans la prochaine section). Ces deux aspects y sont en effet en relation étroite, puisque, on l'aura remarqué, il ne s'agit pas dans cette situation de savoir si un emprunt se justifie ou non: les deux termes en cause sont des emprunts et il convient de choisir celui des deux qui correspond le mieux à l'usage actuel, à savoir falaoa.

On trouvera d'autres exemples de glissement de sens dans un ouvrage récent, dû à Ernst Wendland, The Cultural Factor in Bible Translation ${ }^{9}$. L'auteur a été conseiller en traduction pour la Bible en chichewa (Malawi) et celle en chitonga (Zambie). Il a donc travaillé sur le terrain et ses réflexions se fondent sur une solide expérience. À propos du sujet qui nous intéresse, il dit ceci :

Lorsqu'on procède à des emprunts, il peut y avoir parfois des glissements de sens significatifs. Ainsi, paska (chichewa, emprunt au latin), pour Pâque(s), a été couramment appliqué par les gens de manière spécifique au dernier repas du Seigneur, la Cène (il s'agit là d'un glissement métonymique)... Ou bien Aroma (chichewa, emprunt à l'anglais), pour Romains, est appliqué habituellement aux catholiques romains. Ou encore, kenturiyo (chichewa, emprunté au latin par l'intermédiaire de l'anglais), pour centurion, a été fréquemment compris comme un nom propre, particulièrement dans des contextes tels que celui de Matthieu, 8, 5. 8... C'est pourquoi le traducteur doit contrôler soigneusement tous les mots d'emprunt qui sont utilisés, afin de s'assurer que les gens en général puissent les comprendre correctement dans le contexte où on les emploie.

Un peu plus loin, le même auteur met en garde contre le fait qu'un mot d'emprunt risque, une fois translittéré, d'être semblable à un mot indigène ou à un autre mot précédemment emprunté. Il cite le cas de bbaali (chitonga, de l'anglais barley, orge) qui a été transcrit de la même manière que burley, de l'expression burley tobacco, ce qui est une cause de malentendu évidente. Mais en voilà assez dit pour convaincre chacun de la nécessité d'être attentif aux risques de glissement de sens lorsqu'on procède à un emprunt. Il est temps maintenant de passer à la section suivante, traitant de la question de l'usage.

\section{EMPRUNT ET USAGE}

Comme je l'ai déjà indiqué plus haut, l'emprunt a souvent été une solution de facilité pour des traducteurs de la Bible ne trouvant pas immédiatement dans la langue d'arrivée l'équivalent d'un terme de la langue-source. Cette solution a été très fréquemment appliquée dans le passé, de manière hasardeuse, et, malheureusement, on en rencontre emcore maintes traces dans des versions récentes. On ne s'est pas soucié de vérifier comment le destinataire de la traduction réagirait face à un mot nouveau qui, dans la majorité des cas, lui est inconnu.

Afin de bien orienter la réflexion à ce sujet, il convient de distinguer deux cas principaux : d'une part, il y a le cas où le mot d'emprunt a un sens zéro pour le destinataire ou appartient à un certain jargon théologique, ce qui ne vaut souvent guère mieux; d'autre part, il y a le cas où le mot emprunté est déjà connu et convient parfaitement dans la traduction, même mieux qu'un mot de la langue réceptrice (qui peut être archaïque ou partiellement inadapté au contexte biblique). 


\section{a) Mots à sens zéro ou jargon théologique}

Le théologien suisse Jacques Nicole a consacré récemment un important ouvrage à l'histoire de la traduction de la Bible en tahitien ${ }^{10}$. On y voit comment, au siècle passé, des missionnaires anglo-saxons ont entrepris cette traduction et l'on est frappé de constater la fréquence avec laquelle ils ont recouru à l'emprunt (principalement à partir du grec ou de l'hébreu). L'auteur en donne de multiples exemples ${ }^{11}$. Comme il envisage son sujet sous un angle essentiellement historique, il n'évalue pas cette méthode d'un point de vue critique. Il se borne à mentionner les réactions de ceux qui, assez tôt déjà, ont déploré le nombre excessif de néologismes dans cette version ou qui, plus tard, ont exigé une meilleure exploitation des ressources de la langue locale; ainsi, p. 221 :

L'accroissement rapide du vocabulaire bilingue d'origine étrangère inquiétait d'ailleurs Darling [...] en 1829 [...] De son côté William Howe, qui élimina de la Bible bon nombre de néologismes, profita de la révision de 1847 pour en établir une liste exhaustive [...]»; ou bien, p. 275 : « l'Église évangélique autonome n'était plus d'accord qu'un théologien européen lui retraduise sa Bible [...] elle ébauchait alors un retour aux sources [...] qui la conduisait, en particulier, à revaloriser sa langue et à faire toujours plus confiance à ses propres ressources».

Ces réactions se justifiaient d'autant plus que la plupart des mots empruntés n'avaient aucun sens pour les populations en cause. En effet, il était impossible à celles-ci de saisir la signification de mots comme medebara (forgé sur l'hébreu midebar, désert), mehula (calque de l'hébreu meholah, danse) utilisé dans des contextes d'exultation liturgique, satauro (du grec stauros, croix), ou encore buka (de l'anglais book, livre), etc., (op. cit., respectivement pp. 166, 202 et 156). C'est ainsi qu'on introduisait dans la Bible une quantité de termes à sens zéro, parce qu'on estimait que, dans ces cas-là, le vocabulaire original était intraduisible. Bien entendu, il fallait ensuite fournir un gros effort pour expliquer aux gens de quoi il s'agissait !

À l'époque actuelle, il se trouve encore fréquemment des biblistes qui sont plus ou moins affligés de ce travers. Par exemple, dans la TOB (Traduction œecuménique de la Bible), le traducteur de l'évangile de Jean a cru bon de rendre le terme grec paraklêtos, utilisé à propos du Saint-Esprit, par Paraclet (Jean 14, 16 le Père... vous donnera un autre Paraclet et, v. 26, le Paraclet, le Saint-Esprit; cf. 15,26; 16,7). Quiconque connaît le grec n'aura pas de peine à interpréter le sens de paraklêtos (qu'on peut traduire dans ce cas précis par défenseur, soutien). Les premiers lecteurs du quatrième Évangile pouvaient aisément le comprendre, tandis que pour le commun des mortels, aujourd'hui, Paraclet est une expression dotée d'un mystère insondable qu'elle n'avait pas à l'origine. Et là, on touche directement au problème du jargon théologique cher à d'aucuns, qui se refusent à rendre certains concepts bibliques autrement que par des mots hébreux ou grecs translittérés. C'est ainsi que j'ai pu lire cette phrase dans le bulletin d'une faculté de théologie africaine: «Il apparaît nettement que cette théologie doit avoir comme compléments et prolongements naturels le kerugma, la kö̈nonia et la diakonia en tant que témoignage de la présence de l'Église dans le monde auprès des déshérités...» L'auteur de cette déclaration était sans doute la victime d'un microbe inoculé par les théologiens européens, et cela l'empêchait de parler tout bonnement de «prédication», «communion» et «service».

C'est parce que j'avais en vue de telles aberrations que j'ai écrit dans mon livre Traduire sans trahir: «Dans la grande majorité des cas, le mot d'emprunt est une très mauvaise solution : c'est un mot à sens zéro pour les gens...12 ${ }^{12}$ En réalité, il conviendrait d'être plus nuancé, puisque le mot d'emprunt se justifie toutes les fois où il est consacré par l'usage et où son emploi n'engendre pas de malentendu, comme nous allons le voir pour terminer. 


\section{b) Emprunts justifiés par l'usage}

En ce qui concerne cet aspect de la question, il vaut la peine de se pencher sur le cas du swahili, langue véhiculaire répandue en Afrique centrale et orientale. Il est suggestif à un double titre: 1 . en raison des racines sémitiques qui le caractérisent. Ainsi, Vincent Monteil a pu écrire que «les emprunts du vocabulaire constituent, avec l'écriture, la manifestation la plus saillante de l'arabisation des langues qui, comme le haoussa ou le swahili, ont près de la moitié de leur lexique constituée par des emprunts à la langue arabe ${ }^{13}$ "; 2 . le cas du swahili est intéressant, en second lieu, du fait qu'il a été lui-même l'objet d'emprunts de la part d'autres langues. En travaillant avec des traducteurs du Rwanda et du Burundi, il m'est arrivé à maintes reprises de reconnaître dans leurs textes en kinyarwanda ou en kirundi (deux langues très proches) des mots empruntés au swahili, bien connu dans la région: je les repérais précisément grâce à leurs racines sémitiques. Ces emprunts s'inséraient normalement dans la traduction, car ils étaient d'usage courant et ne risquaient pas, par conséquent, de susciter des difficultés particulières pour les utilisateurs d'une nouvelle version biblique.

Cependant, d'autres influences que celle de l'arabe se font sentir dans le swahili, en particulier celle de l'anglais ou du français, suivant les pays. Vincent Monteil parle à ce propos de «la romanisation qui gagne beaucoup de terrain ${ }^{14} »$. De son côté, Timothy Wilt, un Américain ayant séjourné à Bukavu (est du Zaïre) pendant un certain temps, a relevé l'importance des emprunts au français dans le swahili courant de cette région. À propos d'une nouvelle traduction de la Bible en cette langue, il mentionne les mots empruntés directement au français et conservant l'orthographe française, ou ceux venant de ce qu'il appelle un swahili francisé. Ainsi, on utilisera des mots comme «mètre» ou «kilomètre» pour des mesures de longueur, ou bleu (la couleur) au lieu d'un terme classique, samawi, actuellement inconnu pour la plupart des Zä̈rois. De même, on en est venu à opter pour tribunali (du français «tribunal») de préférence à baraza kubwa: «en effet, dit Wilt, les deux mots baraza et kubwa sont compréhensibles en eux-mêmes, mais, mis ensemble, ils ne sont pas aussi précis que tribunali, terme emprunté au français», qui correspond à une donnée précise dans l'esprit des gens ${ }^{15}$.

Il ressort clairement de ces remarques que: 1. des mots d'emprunt passés dans l'usage courant sont admissibles dans la traduction; 2. certains de ces mots sont actuellement mieux compris de la population en cause que des expressions ayant un sens équivalent dans la langue mais dont le défaut est d'être devenues archaïques. Dans son ouvrage Comment traduire la Bible, Eugene Nida adressait déjà la recommandation suivante aux traducteurs : «Le traducteur évitera de chercher à substituer un mot indigène dont le sens n'est pas immédiatement apparent à un mot d'emprunt qui est d'usage courant et qui est compris $^{16} . \gg$ On voit donc, une fois de plus, que l'utilisation de mots d'emprunt exige une bonne connaissance des divers aspects du problème et des solutions bien pesées, cas pour cas.

\section{CONCLUSION}

Il est temps de conclure. Étant donné tout ce qui a déjà été dit, je peux le faire brièvement.

Les divers exemples que j'ai présentés m'amènent à formuler trois principales remarques:

1. L'abus de l'emprunt dans certaines traductions bibliques a tenu en bonne partie au fait que leurs auteurs étaient souvent des étrangers ayant une connaissance insuffisante des ressources de la langue. Il est donc essentiel, commme on l'exige depuis plusieurs décennies dans le domaine de la traduction biblique, que la traduction de base soit faite par des gens du pays, en leur propre langue, avec, si nécessaire, l'appui de spécialistes venus d'ailleurs. 
2. Mais cet abus tient aussi à l'ignorance de certaines données linguistiques (au sens général de la linguistique, science descriptive du fonctionnement des langues). On part de l'idée que les mots clés sont intraduisibles, parce qu'on les isole de leur contexte particulier et qu'on voudrait leur trouver un équivalent comportant toutes les acceptions qu'ils ont dans divers contextes. Parce que c'est impossible, on recourt à l'emprunt. Il conviendrait donc d'avoir une idée plus précise de la manière dont les mots s'insèrent dans le contexte qui en éclaire l'acception particulière. Dans bien des cas, cette insertion dans le contexte permet de résoudre le problème de la transmission du sens sans avoir à procéder à un emprunt (une certaine terminologie technique mise à part).

3. Enfin, j'ai insisté sur le rôle que doit jouer l'usage pour justifier ou non le choix d'un mot d'emprunt. Mais il est évident que la question est plus facile à régler théoriquement que pratiquement en raison de la tension qui existe partout entre novateurs et puristes ou tenants de la tradition. Cependant, comme je l'ai dit au début de cet exposé, mon propos ne visait pas à arbitrer ce genre de conflit. Mon ambition était simplement d'apporter quelque lumière sur un sujet qui intéresse tout traducteur.

\section{NOTES}

1. Le Nouveau Testament de notre Seigneur Jésus-Christ, traduit sur l'original par une société de ministres de la Parole de Dieu, Lausanne, 1839.

2. BURNIER, L. (1866) : La Version du Nouveau Testament dite de Lausanne. Son histoire et ses critiques, Lausanne.

3. Op. cit., pp. 80 et ss.

4. Linguistique (1980), publié sous la direction de Frédéric François, Paris. Les exemples cités se trouvent pp. 227 et ss.

5. D’après le Dictionnaire latin-français de Félix Gaffiot, Paris, 1934.

6. Voir J.-M. Babut, «La traduction littérale: une statue aux pieds d'argile», META, 32-1, Montréal, mars 1987, pp. 26-29.

7. Voir J.-C. Margot, «La Bible qui divise, la Bible qui rapproche», Perspectives missionnaires, 12, Bevaix (Suisse), 1986, pp. 5-18 (spécialement pp. 13 et ss.).

8. BRADSHAW, J. (1967): «Some New Testament Key Words in Samoan», The Bible Translator, 18-2, Londres, avril 1967, p. 82. Le mot d'emprunt areto a été également utilisé dans la Bible en tahitien (voir dans l'ouvrage cité à la note 10 , ci-dessous, la p. 156).

9. WENDLAND, Ernst R. (1987): The Cultural Factors in Bible Translation, Stuttgart, United Bible Societies, p. 68.

10. NICOLE, J. (1988): Au pied de l'Écriture. Histoire de la Bible en tahitien, Papeete.

11. Voir également Paul HUGUENIN, Raiatea la sacrée, (Neuchâtel, 1902), Papeete 1987, pp. 186 et 209 , n. 1.

12. MARGOT, J.-C. (1979) : Traduire sans trahir, Lausanne, p. 71, cf. p. 246.

13. Ethnologie régionale, volume publié sous la direction de Jean Poirier, Paris, 1972, vol. I, p. 173.

14. Ibid.

15. WILT, T. (1989) : «Two Zairean Swahili Bibles : Dealing with Diglossic Distances», The Bible Translator, 40-3, Londres, juillet, p. 327.

16. NIDA, Eugene A. (1967) : Comment traduire la Bible, Londres, p. 119 (traduction et adaptation française de Bible Translating, $2^{\circ}$ édition révisée, Londres, 1961). 